\title{
Efectos de un programa de ejercicios de control postural en el equilibrio corporal y precisión de lanzamiento en tiro con arco en categoría infantil y cadetes \\ Effects of a postural control exercise program on body balance and accuracy of throwing in archery in children and adolescents \\ *Giovanni Francisco Francino Barrera, **Sergio Rodrigo Jiménez Torres, *Camila Constanza de Jesús Coloma Díaz, *Diana Carolina Delgado Vásquez, *Daniela Francisca Verdugo Millar \\ *Universidad Santo Tomás (Chile), **Universidad de Atacama (Chile)
}

Resumen: El objetivo de este estudio fue el determinar los efectos de un programa de ejercicios de control postural sobre el equilibrio estático y la precisión de lanzamiento del tiro con arco en atletas de la serie infantil (12-14 años) y cadetes (15-17 años). Participaron 12 deportistas para cada serie. Se realizó la prueba de medición de equilibrio a través del Standing Stork Test (ojos abiertos/cerrados) y de la prueba asertividad en la precisión de lanzamiento (6 rondas) en el blanco previo y posterior a la intervención de ejercicios. Ésta última consistió en un programa de entrenamiento de equilibrio bi/unipodal durante 8 semanas. Los resultados mostraron un aumento significativo del equilibrio unipodal con ojos abiertos del grupo infantil en ambas piernas; pero sin cambios en la modalidad de ojos cerrados. La serie cadetes arrojó una diferencia significativa en la prueba de equilibrio unipodal con ojos abiertos sólo en la pierna dominante (derecha); sin cambios en la pierna izquierda, como tampoco diferencias en la modalidad ojos cerrados. La prueba de asertividad del tiro con arco sólo mejoró en el grupo infantil, pero no en la serie cadetes post intervención. Es posible que el entrenamiento de equilibrio postural estático haya optimizado patrones de control postural y visomotores en edades tempranas (bajo los 15 años) en comparación al grupo de cadetes, para así mejorar la precisión del tiro. Se recomienda el potenciar estos ejercicios en edades tempranas para fomentar las destrezas propias del deporte de tiro con arco.

Palabras claves: Tiro con arco, control postural, equilibrio estático, precisión del lanzamiento, niños, adolescentes.

Abstract: The objective of this study was to determine the effects of a postural control exercise program on static balance and accuracy of archery throwing in infant (12-14 years old) and cadet (15-17 years old) categories. A total of 12 athletes per each category took part in the study. As measurement tests, we used the Standing Stork Test (open / closed eyes) for balance and the assertiveness in target launch precision test (6 rounds) before and after the exercise intervention. This consisted of a bi / unipedal balance training program lasting 8 weeks. The results showed a significant increase in unipedal equilibrium with open eyes in the infant group and for both legs; however, no changes were detected in the closed-eye modality. The cadet category showed significant differences in the unipedal equilibrium test with open eyes only for the dominant leg (right); no changes were found for the left leg, nor in the closed-eye modality. The assertiveness test of the archery only improved in the infant group, whereas in cadets it did not change at post-test. It is possible that static postural balance training optimized patterns of postural and visuomotor control at early ages (under 15 years old) compared to the group of cadets, and therefore the throwing accuracy was enhanced. It is recommended to endorse these exercises at early ages so to empower the skills needed in the sport of archery.

Key words: Archery, postural control, static balance, launch accuracy, children, teenagers.

\section{Introducción}

El tiro con arco se describe como un deporte de postura estática que implica una secuencia estable de los patrones de movimiento para producir el lanzamiento de la flecha (Ahmad et al., 2013) (Ahmad, Taha, Hassan, Hisham, Johari et al., 2013). Esta destreza requiere control de movimiento fino, resistencia adecuada, fuerza de la parte superior del cuerpo y la capacidad de equilibrio (Squadrone \& Rodano, 1994)(Squadrone y Rodano, 1994). El disparo en tiro con arco se puede resumir en dos etapas: la visualización del arco; y apuntar/liberar la flecha hacia un objetivo (Ertan, Knicker, Soylu y Strüder, 2011). El control corporal en este deporte es fundamental para mantener una posición bipodal sobre una base fija y es fundamental para el control y dominio de la técnica (Amblard, Cremieux, Marchand y Carblanc, 1985). El óptimo control de la postura en la arquería se relaciona con la mayor habilidad que posee una/un deportista estabilizando el equilibrio justo antes de disparar la flecha(Hrysomallis, 2011). Este control requiere una postura erguida constante frente a la preparación de un movimiento

Fecha recepción: 15-02-19. Fecha de aceptación: 19-09-19 Giovanni Francisco Francino Barrera

giovannifrancinoba@santotomas.cl voluntario, conservando así energía para dar mayor precisión del funcionamiento del tren superior antes y durante la ejecución del tiro (Deliagina, Zelenin, Beloozerova y Orlovsky, 2007).

El uso de ajustes posturales para la estabilidad corporal es dado a través del sistema nervioso central (SNC) mediante un óptimo procesamiento sensorial y respuesta de los efectores somáticos. Se requiere de un adecuado procesamiento del sistema visual, vestibular y somatosensorial (Crossman, 2007). Si bien la postura puede estabilizarse a través de ajustes automáticos por al arco reflejo a nivel medular, el SNC también puede iniciar ajustes posturales anticipatorios frente a las perturbaciones voluntarias (Belenkii, Gurfinkel y Paltsev, 1967). En el caso de la arquería, la ejecución del tiro empleando los brazos requiere ajustes preprogramados a nivel de tronco y extremidades inferiores para mantener una postura estable y optimizar el tiro. Debido a que estos ajustes posturales son iniciados antes de la perturbación voluntaria de la postura, se argumenta entonces que estos son preprogramados por el SNC (Bouisset y Zattara, 1981). Estos ajustes posturales se ven alimentados por las aferencias sensoriales, principalmente las oculares.

Las aferencias visuales poseen un importante papel en los movimientos combinados ojos-cabeza-cuello de tal for- 
ma que existe una estrecha relación entre la visión, sistema vestibular y la propiocepción muscular del cuello para el mantenimiento postural (Bove, Fenoggio, Tacchino, Pelosin y Schieppati, 2009). El sistema visual proporciona información sobre el medio circundante, distancia del cuerpo con objetos externos y respecto al terreno en que se produce el movimiento corporal o de extremidades (Umphred, Byl, Lazaro y Roller, 2001). Es por esto que, el entrenamiento deportivo basado en ejercicios propioceptivos mejora la capacidad de usar información somatosensorial, visual y otolítica para optimizar la postura (Amblard y Carblanc, 1980; Bove et al., 2009). El tiempo de respuesta visual y la coordinación ojocuerpo son las habilidades más importantes en el tiro con arco (Ahmad et al., 2013; Carlson, 1985). Esta habilidad está involucrada en la capacidad de la/del atleta para ajustar su equilibrio en respuesta a un estímulo visual ((Loran y MacEwen, 1995). Como la precisión del tiro con arco estaría relacionada con la reducción de la oscilación corporal justo antes de soltar la flecha (Hrysomallis, 2011; Mason y Pelgrim, 1986), la información lineal, juegan un papel indispensable en la orientación y la estabilidad postural (Amblard et al., 1985; Calavalle et al., 2008) para esta disciplina. Sin embargo, las otras aferencias tales como la propiocepción también son indispensables para estabilizar al cuerpo cuando se realiza una coordinación ojo-cabeza-cuello y cuerpo.

En este estudio nos propusimos medir la influencia del entrenamiento basado en balance corporal sobre el equilibrio estático y, por consiguiente, en la precisión del lanzamiento en tiro con arco en series infantiles y cadetes. Esto debido a que las habilidades motrices pueden ser mejor adaptadas y potenciadas antes y durante el proceso de maduración del control del equilibrio corporal. Por otro lado, la mayoría de este tipo de deportistas, en la localidad del estudio inician su carrera desde niños (Paulus, Straube, Krafczyk y Brandt, 1989). Muchos deportistas adultos de alto rendimiento demuestran mejor control del equilibrio corporal y asertividad en el tiro del arco, en comparación con personas aficionadas (Gagey y Weber, 2001; Paulus et al., 1989), lo que podría explicarse debido a la no sólo a la temprana inclusión al ejercicio de esta disciplina, sino que también al tiempo de entrenamiento dedicado al balance corporal y a la precisión para obtener mejores marcas deportivas en ella. Es por esto que, el objetivo de este estudio fue determinar los efectos que tendrían los ejercicios propioceptivos de control postural en el equilibrio corporal y precisión de lanzamiento en tiro con arco en arqueras/os en categoría infantil y cadete. Se hipotetizó que el entrenamiento por balance estático mejoraría el equilibrio corporal y precisión de lanzamiento en ambas categorías.

\section{Metodología}

\section{Participantes}

La muestra de este estudio fue de un grupo de 12 participantes en categoría infantil (de 12 a 13años) y otras/os 12 de la categoría cadetes mixtas (de 15 a 17 años). El grupo infantil estaba conformado de 5 mujeres y 7 hombres, mientras que la serie de cadetes por 7 mujeres y 5 hombres. Todos ellos informaron poseer lateralidad óculo-mano-pie derecha. La media y desviación estándar (DS) del peso de la serie infantil fue de $44,1 \pm 3,4$ y para la de cadetes fue de $66,5 \pm 5,7 \mathrm{~kg}$. La media y DS para la talla fue en la categoría infantil de 156,2 $\pm 4,8$ cm, y la de cadetes fue de $167,1 \pm 8,4 \mathrm{~cm}$. Ambos grupos fueron evaluados de manera separada comparando el pre y post test de una intervención basada en ejercicios de control postural. El consentimiento informado fue entregado a cada participante para ser firmado por sus progenitores o tutores legales. Este consentimiento fue revisado y aprobado por la Junta de Revisión Institucional de la Universidad (CEC UST $N^{\circ}$ 21/2017). El diseño de este estudio fue pre-experimental a través de un pre y post test comparando las variables de equilibrio estático y la precisión de aciertos en el blanco (diana) en deportistas de ambas categorías mencionadas arriba pertenecientes a un club de tiro con arco tipo compuesto.

\section{Instrumentos.}

Las evaluaciones consistieron en evaluar el equilibrio estático y la precisión de lanzamientos en la diana. La prueba de equilibrio estático se realizó mediante el Standing Stork Test «(SST, ver figura 1)». En esta prueba se midió con un cronómetro la cantidad de tiempo en segundos (s) que el participante puede mantenerse en la posición unipodal con ojos abiertos y ojos cerrados, lo cual, es indicativo de su rendimiento de equilibrio (Maki \& McIlroy, 1997)(Maki y McIlroy, 1997). Posteriormente se evaluó la precisión mediante la prueba de asertividad en lanzamiento de 6 rondas (TAL6R) según el round FITA(Federación Internacional de Tiro con Arco) a 70 metros de distancia en campo abierto (ver figura 2) (Cabo Jiménez, et al., 2012, Haywood \& Lewis, 2013, Márquez \& Carracedo, 2016)(Cabo Jiménez, Álvarez Fernández y Estrada Contreras, 2012; Haywood y Lewis, 2013; Márquez y Carracedo, 2016). Se realizaron 10 tiros por

Figura 1. Standing Stork Test
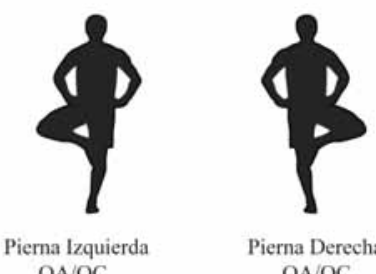
$\mathrm{OA} / \mathrm{OC}$

Figura 1. Prueba de equilibrio estático pretest y postet basado en el Standing Stork Test donde los participantes debían mantener el equilibrio en un solo pie con el otro apoyado en su rodilla y manos en la cintura, por el mayor tiempo posible sin perder la estabilidad (s).

Figura 2. Campo de tiro

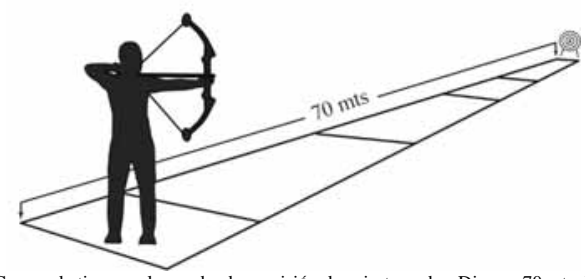

Figura 2. Campo de tiro para la prueba de precisión de aciertos sobre Diana a 70 mts de distancia. Figura 3. Diana oficial estilo FITA

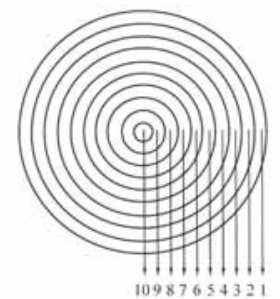

Figura 3. Diana oficial para la ejecución de tiros según puntaje del 1 al 10 en prueba de aciertos. 
ronda, contabilizando un total de 60 tiros por participante. La diana oficial estilo FITA consistió en un blanco redondo de 10 anillos donde para el más céntrico, se obtenía una puntación de 10. A medida que la flecha se alejaba del centro de la diana, iba perdiendo la puntuación (ver figura 3) (Cabo Jiménez et al., 2012).

El entrenamiento de control postural consistió en una progresión de ejercicios de equilibrio estático bipodal y unipodal con ojos abiertos (OA) y cerrados (OC) en 8 semanas, 3 sesiones por semana para ambos grupos(ver figura 4). Por último, este estudio analizó las medias y desviación estándar de las variables de equilibrio estático, y de la cantidad de aciertos, que se obtuvieron de la evaluación pre y post intervención en ambas categorías por sí solas. Estos datos fueron comparados mediante la prueba estadística $\mathrm{F}$ de Fisher a través del análisis de datos con el software estadístico SYSTAT,versión 12 (Systat Software, Inc. Chicago, IL, USA).

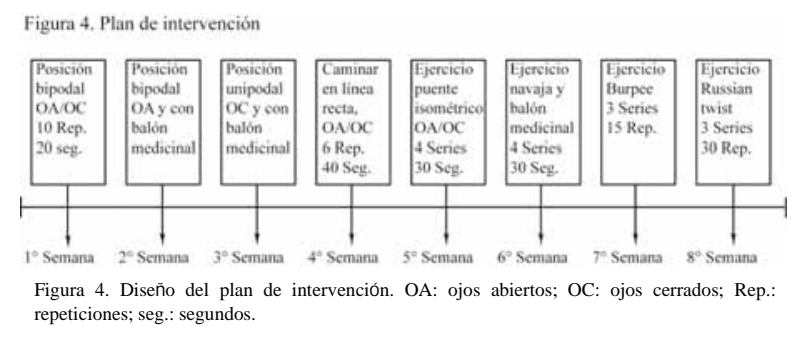

\section{Resultados y discusión}

\section{Standing Stork Test en categoría infantil (de 12 a 14 años)}

La tabla 1 muestra todos los valores de las medias y desviaciones estándar de todas las pruebas analizadas y el nivel de significancia. La gráfica 1 muestra las medias y DS de los tiempos de duración del equilibrio unipodal según el SST en la evaluación inicial y final tanto para OA como para la OC en la categoría infantil. En la condición de SST con OA hubo una diferencia significativa en la duración de la postura de equilibrio unipodal en ambas piernas después de la intervención, de al menos $80 \mathrm{~s}$ al inicio, terminando alrededor de unos $155 \mathrm{~s}$, al final del entrenamiento (ambas $\mathrm{p}<0,05$ ). Sin embargo, para condición del SST con OC no hubo diferencias, manteniéndose bajas en los dos períodos de tiempo. Los resultados significativos de incremento del control postural unipodal, para ambas piernas podrían deberse al aumento súbito que experimentan los niños durante los 12 a 14 años (Nelson, 1999). Es sabido que la organización sensorial para la postura demora en desarrollarse alcanzando el óptimo desarrollo por encima de los 15 años (Hirabayashi y Iwasaki, 1995). Sin embargo, esto contrasta con nuestros resultados, donde, el tratamiento de ejercicios de control postural mejora el proceso de adaptación a mantener posturas, después de la intervención de ejercicios. , Estos resultados en en la condición de SST con OA podrían deberse a la falta de maduración de asimetría de la lateralidad (Mikheev, Mohr, Afanasiev, Landis y Thut, 2002) donde ambas piernas de las/los atletas se comportaron de casi idéntica manera en aumentar la postura unipodal. Al analizar el SST con OC, donde no hubo diferencias entre el pretest y postest, se podría deber por otro lado a la alta dependencia de la aferencia visual, (Amblard y Carblanc, 1980; Bove et al., 2009; Umphred et al., 2001) para la coordinación oculomotora, aun por la falta de desarrollo de la neuroplasticidad en esta edad.

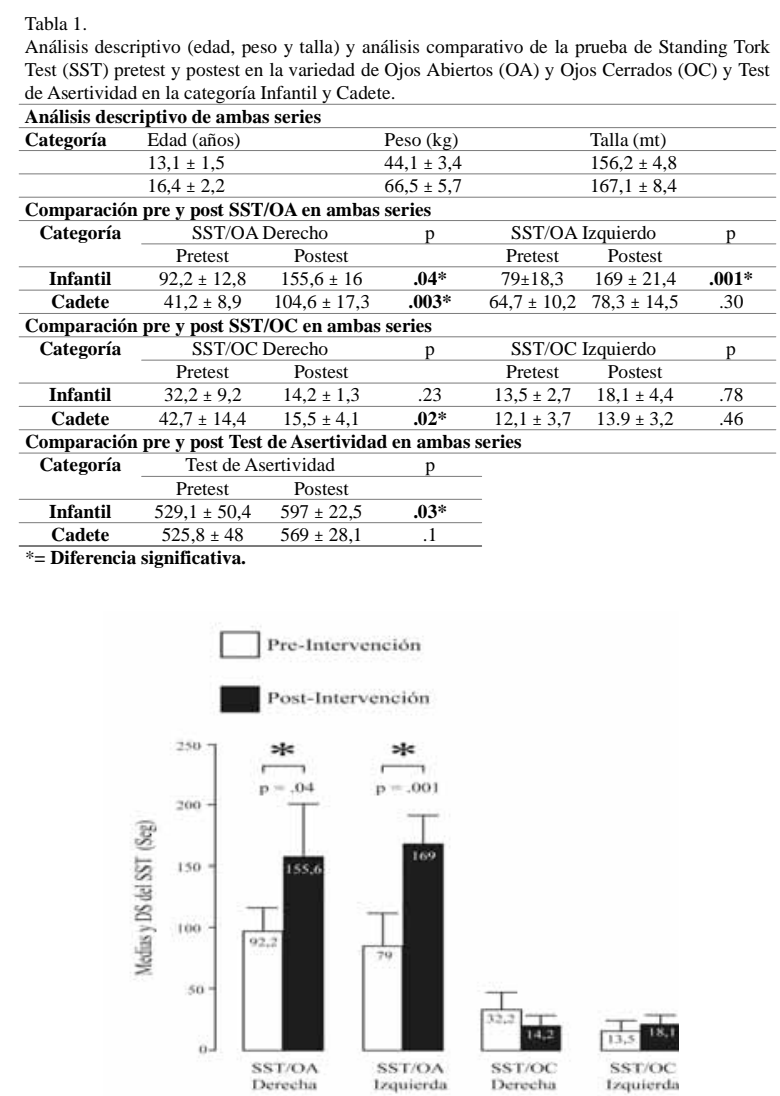

Gráfica 1. Diferencias entre el Standing Stork Test (SST) para apoyo unipodal izquierdo y derecho con ojos abiertos (OA) y cerrados (OC) antes y después de la intervención en el grupo Infantil.

\section{Standing Stork Test en categoría cadete (de 15 a 17 años)}

La gráfica 2 muestra las medias ydesviaciones estándar de los tiempos de equilibrio estático según el SST en la evaluación inicial y final para la condición de OA y OC en la categoría cadetes. Los resultados muestran un cambio significativo del rendimiento en la condición OA del SST en la pierna derecha $(\mathrm{p}<.05)$ de 41,2 a $104,6 \mathrm{~s}$; pero no para la pierna izquierda 64,7 en el pretest frente a los 78,3 s en el postest ( $\mathrm{p}>$.05). Esto explicarse por la maduración de la asimetría de control (Mikheev et al., 2002) donde a la edad de 15 a 17 años, la lateralidad dominante (en este caso, la pierna derecha) está mayormente desarrollada (Batez et al., 2018; Gonzalez-Jurado, Boza, Vázquez, Bendala y Otero-Saborido, 2016). Esta estrategia se explica por la preferencia a un miembro inferior (Lamm, Zelazo y Lewis, 2006) y dirección de esta elección (Armitage y Larkin, 1993) para distribuir mejor la cargas y ajustes posturales, como desarrollo del control postural observada en adultos sanos (Bettega, Scaglia, do Nascimento, Godoy y Galatti, 2018; Mikheev et al., 2002). Por otro lado, se observa un descenso significativo de la pierna derecha pierna derecha resultó en una disminución en los tiempos de la mantención de postura unipodal durante la condición SST con OC (de 45 a 29 s; p<.05). Esto podría deberse a la dependencia a la aferencia visual sería un factor importante ante la ausencia de una orientación del cuerpo en 
el espacio (Amblard y Carblanc, 1980; Loran y MacEwen, 1995). Por otra parte, la pierna izquierda mantuvo un tiempo similar en condición OC del SST en ambos períodos. Esto evidenciaría aún más la gran dependencia al estímulo visual (Crossman, 2007; De la Fuente y Gómez-Landero Rodríguez, 2019) y asimetría de lateralidad (Bravo, Rodríguez-Negro y Irigoyen, 2017; Guedes, Franzini, Júnior y Morales, 2017; Mikheev et al., 2002) para arqueros cadetes para pararse en la pierna menos diestra.

\section{Test de asertividad de tiro con arco serie infantil}

En la gráfica 3 muestra la diferencia de las medias y desviación estándar de la prueba de asertividad con que los arqueros infantiles obtuvieron al tiro de flecha desde el inicio y posterior a la intervención. Se observa una mejoría notable desde un puntaje de 525 a uno de 597, con una diferencia significativa de 76 puntos $(\mathrm{p}<.05)$. Esto demuestra

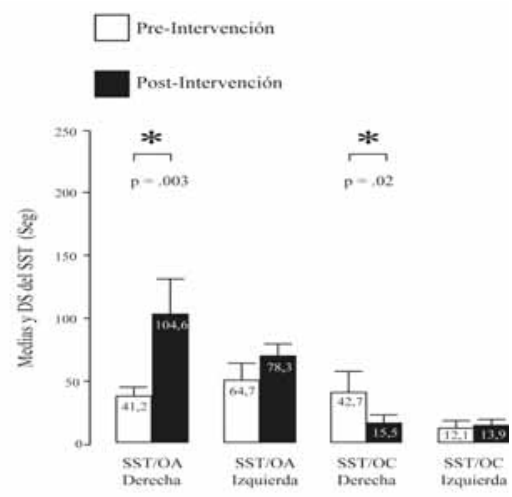

Gráfica 2. Diferencias entre el Standing Stork Test (SST) para apoyo unipodal izquierdo y derecho con ojos abiertos $(\mathrm{OA})$ y cerrados $(\mathrm{OC})$ antes y después de la intervención en el grupo Cadete.

la eficacia del entrenamiento de equilibrio, gracias a sus virtudes de mejora sensorial de la postura (Amblard y Carblanc, 1980; Bove et al., 2009) en conjunto con el aumento de la fuerza muscular (González, Moreno-Arrones, Bretones y de Villarreal Sáez, 2018). Por otro lado, también esta mejora de la eficacia de tiro con arco en esta serie podría deberse a una mejor concentración y disciplina (Sáez-Gallego, VilaMaldonado, Abellán y Contreras, 2018), mayor dedicación a la habilidad deportiva (Batez et al., 2018), optimización del proceso de afianzamiento de la habilidad (Bravo et al., 2017; Clemente, Moreno-Arrones y Gil, 2019), maduración física (De la Fuente y Gómez-Landero Rodríguez, 2019; Guedes et al., 2017), entre otras. También la mejora en la precisión de tiro podría explicarse por a la optimización de otra variable como la agudeza visual, la cual podría no sólo ayudar a mejorar el equilibrio, sino también a las habilidades visomotoras (Batez et al., 2018; Lee, Chiu, Liu y Lin, 2009). Esto contrasta con la idea de muchos entrenadores en el área, donde prefieren exigir mayor destreza deportivas en etapas de mejor coordinación corporal, por sobre el peak del control de ajustes posturales (Hirabayashi y Iwasaki, 1995), es decir, sobre los 15 años.

\section{Test de asertividad de tiro con arco serie cadete}

La gráfica 4 muestra la media y desviación estándar del puntaje de precisión antes y después del entrenamiento de equilibrio en la serie cadetes.. La diferencia entre la media inicial de 525 y la final de 569, no fue estadísticamente significativa ( $>>$.05).. Estos resultados pueden ser debidos a la

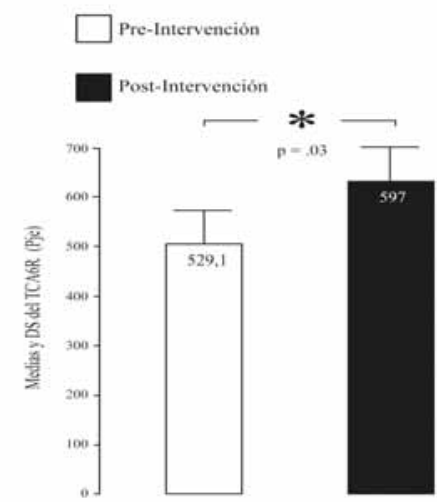

Gráfica 3. Diferencias entre la prueba de asertividad a 6 rondas (TCA6R) antes y después de la intervención en el grupo Infantil.

maduración de los centros que controlan el equilibrio postural (Hirabayashi y Iwasaki, 1995; Nelson, 1999), del sistema visomotor (Loran y MacEwen, 1995), lo que repercutiría en la habilidad de rendimiento del tiro y rondas de tiro, como se observa en los participantes de esta categoría.

Los resultados de este estudio demuestran que los participantes de la serie infantil obtuvieron mejorías en el control postural y fueron más precisos que los cadetes con factores de equilibrio. Podría ser que la técnica de tiro con arco es adquirida a corto plazo en edades tempranas siendo el control del equilibrio el que requiere más precisión en etapas posteriores (Amblard et al., 1985; Bettega et al., 2018; Saraiva et al., 2017). La hipótesis de que el entrenamiento basado en ejercicios propioceptivos tendría una mejoría en el equilibrio y asertividad en serie cadetes, sin embargo, los resultados de este estudio demostraron que esta optimización fue mejor observada en la serie de participantes menores a 15 años.

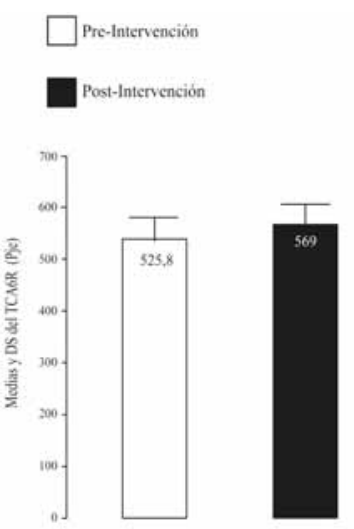

Gráfica 4. Diferencias entre la prueba de asertividad a 6 rondas (TCA6R) antes y después de la intervención en el grupo Cadete.

\section{Conclusiones}

Hubo una mejoría en la habilidad de controlar el equilibrio de ambas piernas en la categoría infantil. Sin embargo, no hubo cambios de esta prueba en la modalidad de ojos cerrados, debido a la alta dependencia de la aferencia visual propia de esta edad. Por otro lado, En la condición del test de equilibrio con OA en deportistas del tiro con arco en la serie de cadetes sólo lograron un aumento significativo del tiempo del equilibrio estático en la pierna derecha y no en la izquierda, posiblemente debido a la maduración de la lateralidad de miembros inferiores. Debido a la dependencia de la entrada visual, la pierna dominante disminuyó el tiempo 
de equilibrio en el SST con OC, mientras que la pierna contralateral no presentó diferencia significativa entre el pretest y postest.

Hubo una diferencia significativa en la precisión del tiro con arco en la categoría infantil, pero no en la de cadetes, ya que la mejora del equilibrio en ambas piernas y de las habilidades visomotoras habrían sido influyentes durante el peak de maduración de ajustes posturales, pero no después de los 15 años, como ocurrió en los arqueros de la categoría cadetes. Por tanto, En el inicio de las habilidades motoras y visuales es donde debería mejorar los rendimientos de este deporte en edades antes de los 15 años, y potenciar los ejercicios de estabilidad corporal para aumentar el rendimiento deportivo de esta disciplina en futuras competencias. .Finalmente, se puede concretar que este estudio respondió parcialmente a la hipótesis de que ambos grupos producirián cambios en la mejora significativa del equilibrio estático y el test de asertividad de tiro, ya que sólo la categoría infantil alcanzó estos logros después de la intervención con ejercicios propioceptivos. Las limitaciones de esta investigación estuvieron centradas en los números de la muestra y en la falta de otras categorías para poder tener un mayor rango de observaciones del comportamiento motor y precisión en otras edades, esto debido a las condiciones de la localidad. Se recomienda para futuras líneas de investigación, aumentar el número de participantes, mayor espectro de edad y/o comparación con otros deportes que incorpores el equilibrio estático para el logro competitivo.

\section{Agradecimientos}

Se agradece al Instituto Nacional del Deporte (IND) de Chile y al Club de Tiro con Arco «Ajayu Thaya» de Arica por el apoyo dado en facilitar a los deportistas en esta investigación. También agradecer a la Escuela de Kinesiología UST Arica por la prestación del Laboratorio de Análisis del Movimiento Humano para llevar a cabo las evaluaciones pertinentes.

\section{Conflicto de intereses:}

Los autores declaran no poseer conflictos de intereses.

\section{Referencias}

Ahmad, Z., Taha, Z., Hassan, H. A., Hisham, M. A., Johari, N. H., y Kadirgama, K. (2013). Biomechanics measurements in archery. En: A. Editor y B. Editor (Eds.), International Conference on Mechanical Engineering Research (ICMER2013), (Vol. 1, p. 3).

Amblard, B., Carblanc, A. (1980). Role of foveal and peripheral visual information in maintenance of postural equilibrium in man. Perceptual and Motor Skills, 51(3), 903-912. Recuperado de https://doi.org/10.2466/pms.1980.51.3.903

Amblard, B., Cremieux, J., Marchand, A., y Carblanc, A. (1985). Lateral orientation and stabilization of human stance: static versus dynamic visual cues. Experimental brain research, 61(1), 21-37.

Armitage, M., y Larkin, D. (1993). Laterality, motor asymmetry and clumsiness in children.Human Movement Science,
12(1-2), 155-177. Recuperado de https://doi.org/10.1016/0167-9457(93)90041-M

Batez, M., Krsmanovic, B., Mikalacki, M., Cokorilo, N., Simic, M., y Montero, P. J. R. (2018). Morphological characteristics and motor skills of young students with different levels of engagement in physical activities. Retos: nuevas tendencias en educación física, deporte y recreación(33), 58-62.

Belenkii, V., Gurfinkel, V., y Paltsev, E. (1967). On the control elements of voluntary movements. Biofizika.

Bettega, O. B., Scaglia, A. J., do Nascimento, J. V., Godoy, S. J. I., \& Galatti, L. R. (2018). O ensino da tática e da técnica no futebol: concepção de treinadores das categorias de base. Retos: nuevas tendencias en educación física, deporte y recreación(33), 112-117.

Bouisset, S., y Zattara, M. (1981). A sequence of postural movements precedes voluntary movement. Neuroscience letters, 22(3), 263-270. Recuperado de https://doi.org/10.1016/0304-3940(81)90117-8

Bove, M., Fenoggio, C., Tacchino, A., Pelosin, E., y Schieppati, M. (2009). Interaction between vision and neck proprioception in the control of stance. Neuroscience, 164(4), 1601-1608. Recuperado de https://doi.org/10.1016/ j.neuroscience.2009.09.053

Bravo, I., Rodríguez-Negro, J., y Irigoyen, J. Y. (2017). Diferencias en función del género en la puntería y atrape en niños de Educación Primaria. RETOS. Nuevas Tendencias en Educación Física, Deporte y Recreación (32), 35-38.

Cabo Jiménez, J. A., Álvarez Fernández, M. Á., y Estrada Contreras, O. (2012). El tiro con arco como contexto deportivo para el fomento de la igualdad entre hombres y mujeres. Paper presented at the Investigación y género, inseparables en el presente y en el futuro: IV Congreso Universitario Nacional Investigación y Género,[libro de actas]. Facultad de Ciencias del Trabajo de la Universidad de Sevilla, 21 y 22 de junio de 2012.(Coord.) Isabel Vázquez Bermúdez;(Com. cient.) Consuelo Flecha García...[et al.](pp. 297-308). Sevilla: Unidad para la Igualdad, Universidad de Sevilla. Recuperado de http://hdl.handle.net/11441/39584

Calavalle, A., Sisti, D., Rocchi, M., Panebianco, R., Del Sal, M., y Stocchi, V. (2008). Postural trials: expertise in rhythmic gymnastics increases control in lateral directions. European journal of applied physiology, 104(4), 643-649.

Carlson, S. J. (1985). A neurophysiological analysis of inhibitive casting. Physical \& Occupational Therapy in Pediatrics, 4(4), 31-42. Recuperado de https://doi.org/ 10.1080/J006v04n04_04

Clemente, J.A.A., Moreno-Arrones, L. J. S., y Gil, S. S. (2019). Diferencias entre distintas orientaciones del espacio, relativizadas al perfil individual del jugador. Retos: nuevas tendencias en educación física, deporte y recreación(35), 3-6.

Crossman, A. (2007). Neary D. Neuroanathomy, Churchill Livingstone, Edinborough.

De la Fuente, A., \& Gómez-Landero Rodríguez, L. (2019). Motor differences in cadet taekwondo athletes according to competition level. Revista Internacional de Medici- 
na y Ciencias de la Actividad Física y del Deporte, 19(73).

Deliagina, T. G., Zelenin, P. V., Beloozerova, I. N., y Orlovsky, G. N. (2007). Nervous mechanisms controlling body posture. Physiol Behav, 92(1-2), 148-154. doi: 10.1016/ j.physbeh.2007.05.023. Recuperado de https://doi.org/ 10.1016/j.physbeh.2007.05.023

Ertan, H., Knicker, A., Soylu, R., y Strüder, H. (2011). Individual variation of bowstring release in high level archery: a comparative case study. Human Movement, 12(3), 273276. Recuperado de https://doi.org/10.2478/v10038-0110030-x

Gagey, P.-M., y Weber, B. (2001). Posturología: regulación y alteraciones de la bipedestación: Elsevier España.

Gonzalez-Jurado, J., Boza, S. R., Vázquez, M. C., Bendala, F. T., y Otero-Saborido, F. M. (2016). Comparación de un entrenamiento propioceptivo sobre base estable y base inestable. Revista Internacional de Medicina y Ciencias de la Actividad Física y del Deporte/International Journal of Medicine and Science of Physical Activity and Sport, 16(64), 617-631.

González, J. R., Moreno-Arrones, L. J. S., Bretones, A. R., y de Villarreal Sáez, E. S. (2018). Efectos a corto plazo de un programa de entrenamiento de sobrecarga excéntrica sobre el rendimiento físico en jugadores de fútbol de élite U-16. Retos: nuevas tendencias en educación física, deporte y recreación(33), 106-111.

Guedes, D. P., Franzini, P. C., Júnior, R. P., y Morales, J. M. M. (2017). Antropometria e Aptidão Física de Adolescentes Latino-Americanos. Retos: nuevas tendencias en educación física, deporte y recreación(31), 264-270.

Haywood, K., \& Lewis, C. (2013). Archery-4th Edition: Steps to Success: Human Kinetics.

Hirabayashi, S.-i., y Iwasaki, Y. (1995). Developmental perspective of sensory organization on postural control. Brain and development, 17(2), 111-113. Recuperado de https://doi.org/10.1016/0387-7604(95)00009-Z

Hrysomallis, C. (2011). Balance ability and athletic performance. Sports medicine, 41(3), 221-232.

Lamm, C., Zelazo, P. D., y Lewis, M. D. (2006). Neural correlates of cognitive control in childhood and adolescence: Disentangling the contributions of age and executive function. Neuropsychologia, 44(11), 2139-2148. Recuperado de https://doi.org/10.1016/ j.neuropsychologia.2005.10.013

Lee, A. J., Chiu, Y.-C., Liu, Y.-F., y Lin, W.-H. (2009). The effect of 8 weeks archery training on postural stability in children. Gait \& Posture, 30, S117. Recuperado de https://doi.org/ 10.1016/j.gaitpost.2009.08.175

Loran, D. F., y MacEwen, C. J. (1995). Sports vision: Butterworth-Heinemann.

Maki, B. E., y McIlroy, W. E. (1997). The role of limb movements in maintaining upright stance: the «changein-support» strategy. Physical therapy, 77(5), 488-507. Recuperado de https://doi.org/10.1093/ptj/77.5.488

Márquez, J. C. S., y Carracedo, J. E. G. (2016). La enseñanza de tiro con arco en la iniciación deportiva (Revisión). Olimpia: Publicación científica de la facultad de cultura física de la Universidad de Granma, 13(41), 49-57. Mason, B. R., y Pelgrim, P. P. (1986). Body stability and performance in archery. Excel, 3(2), 17-20.

Mikheev, M., Mohr, C., Afanasiev, S., Landis, T., y Thut, G. (2002). Motor control and cerebral hemispheric specialization in highly qualified judo wrestlers. Neuropsychologia, 40(8), 1209-1219. Recuperado de https://doi.org/10.1016/S0028-3932(01)00227-5

Nelson, C. A. (1999). Neural plasticity and human development. Current Directions in Psychological Science, 8(2), 42-45. Recuperado de https://doi.org/ 10.1111/1467-8721.00010

Paulus, W., Straube, A., Krafczyk, S., y Brandt, T. (1989). Differential effects of retinal target displacement, changing size and changing disparity in the control of anterior/posterior and lateral body sway. Experimental Brain Research, 78(2), 243-252.

Sáez-Gallego, N., Vila-Maldonado, S., Abellán, J., y Contreras, O. (2018). El entrenamiento perceptivo de bloqueadoras juveniles de voleibol. Revista Internacional de Medicina y Ciencias de la Actividad Física y del Deporte.

Saraiva, A., Borba-Pinheiro, C., Reis, V., da Silva, J. B., Drigo, A., Mataruna-Dos-Santos, L., y Novaes, J. (2017). Orden en los ejercicios de fuerza sobre el rendimiento de los judokas. Revista Internacional de Medicina y Ciencias de la Actividad Física y del Deporte/International Journal of Medicine and Science of Physical Activity and Sport, 17(68), 605-617.

Squadrone, R., y Rodano, R. (1994). Multifactorial analysis of shooting archery. Paper presented at the ISBSConference Proceedings Archive.

Umphred, D., Byl, N., Lazaro, R., y Roller, M. (2001). Interventions for neurological disabilities. Neurological rehabilitation, 4, 56-134.

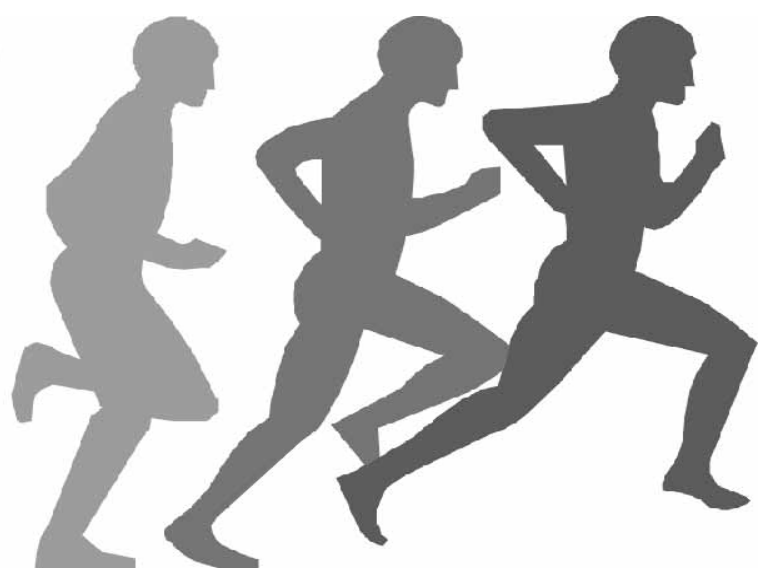

